\title{
Milbenprävention: eine Metaanalyse - Vertrauen gerechtfertigt?
}

$\mathrm{I}$ m Zeitalter der Evidence Based Medicine gilt die Metaanalyse als Gipfel der wissenschaftlichen Bewertungsverfahren. Nunmehr liegt eine Metaanalyse auch zu dem Wert der häuslichen Milbenkontrollmaßnahmen bei Asthma bronchiale vor, immerhin aus dem Nordic Cochrane Centre in Kopenhagen (Gøtzsche et al., BMJ, 1998, 317: 1105-1110).

Die Autoren kommen $\mathrm{zu}$ dem Schluß, daß die gegenwärtigen chemischen und physikalischen Verfahren zur Reduktion der Milbenallergenbelastung ineffektiv sind und nicht als prophylaktische Behandlung bei Milbenasthma empfohlen werden können.

\section{Methodische Mängel}

Die Schlußfolgerung fußt auf der Analyse von 23 von insgesamt 458 erfaßten Studien, die zurückreichen bis ins Jahr 1973. Eingeschlossen wurden so unterschiedliche Verfahren wie Staubsaugen, Benzylbenzoat, elektrostatische Abscheider, Natamycin, Laminar Flow, Luftfilter und Ionisatoren. Wen wundert es, daß das Gesamtergebnis negativ ausfallen mußte? In fünf der Studien wurden Effekte auf die Milben(allergen)belastung überhaupt nicht erfaßt.

Ein weiteres Problem der Metaanalyse beruht auf dem Wirksamkeitsnachweis allein aufgrund der Asthmasymptome und des morgendlichen Peak Flows. So wurde u.a. die sehr überzeugende Arbeit von Ehnert et al. aus dem Jahr 1992, in der die Reduktion der bronchialen Hyperreagibilität belegt wurde, zwar (doppelt(!), sowohl als doppelblinde als auch als offene Studie, mit bzw. ohne Verringerung der Milbenbelastung) erfaßt, aber nicht in die Darstellung der Karenzeffekte einbezogen.

Daß eine Milbenallergenvermeidung bei Milbenasthma effektiv ist, wird aus einer Reihe von Untersuchun- gen bei Kindern während längerer Hochgebirgsaufenthalte geschlossen, obwohl die Besserung auch auf anderen Faktoren als der Milbenarmut über $1500 \mathrm{~m}$ beruhen könnte (Custovic et al., 1998).

Es stellte sich seit langem die Frage, ob eine vergleichbar wirksame Milbenprävention auch im Flachland möglich ist. Frühere Karenzempfehlungen hatten keine wissenschaftliche Basis und waren daher zumeist teuer und wirkungslos.

Erst seit Untersuchungen über die Milbenallergenbelastung des Wohnungsstaubes möglich sind und dadurch die Beziehung zwischen Allergenbelastung und Atemwegserkrankung in zahlreichen Details geklärt werden konnten (Lau et al., 1989; Wahn et al., 1997), ist die Erarbeitung sinnvoller Präventionsstrategien möglich geworden (International Consensus Report 1992 etc.).

Es ist aus diesen Untersuchungen deutlich geworden, daß eine Milbenkarenz nicht nur für die Sekundärprävention von Bedeutung ist, sondern auch zu den wichtigsten Aufgaben der Primärprävention gehören sollte (Peat, Li, 1999).

\section{Studien neueren Datums}

Erst Studien nach neueren Erkenntnissen - und auch mit ausreichender Studiendauer - können vernünftigerweise auf ihre Effektivität hin ausgewertet werden. Die effektivste Milbenprävention ist durch die Verwendung Milbenallergen-undurchlässiger $\mathrm{Ma}-$ tratzen-, Kissen- und Bettbezüge möglich.

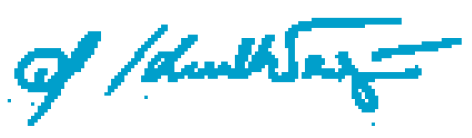

Prof. Dr. G. Schultze-Werninghaus
Es liegen sechs kontrollierte Untersuchungen bei Kindern und Erwachsenen vor, in denen eine signifikante Reduktion von Milben bzw. Milbenallergen erreicht wurde und die lang genug waren, um auch klinische Effekte zeigen zu können (Walshaw, Evans, 1986; Ehnert et al., 1993; Carswell et al., 1996; van der Heide et al., 1997; Halken et al., 1997; van der Heide et al., 1997).

Klinischer Effekt jetzt nachweisbar In diesen Untersuchungen - von denen nur drei in der o.g. Metaanalyse erfaßt sind - wurde jeweils ein klinischer Effekt auf die Asthmasymptomatik gesehen, gemessen an Lungenfunktion, Symptomen oder Medikation; die Effekte auf die bronchiale Hyperreagibilität waren variabel. In weiteren Untersuchungen wurden auch günstige Effekte bei Rhinitis und atopischem Ekzem beobachtet (Übersicht: Custovic et al., 1998).

Neuere Untersuchungen belegen somit, daß eine wirksame Milbenprävention möglich ist und auch zu klinischen Effekten führt. Zweifellos ist die Thematik noch nicht in allen Aspekten abgeklärt, um bereits flächendeckende Maßnahmen empfehlen zu können. Besonderes Augenmerk verdient vor allem die Frage, ob in $\mathrm{Zu}-$ kunft mit einer Reduktion des Milbenallergens in der Wohnung eine wirksame Primärprävention, d.h. eine Verringerung der Asthmaprävalenz, möglich sein wird.

Merke: Vertraue nicht jeder Metaanalyse.

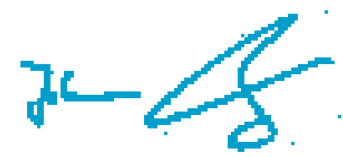

Prof. Dr. Dr. J. Ring 